phylogenetic tree. Part IV of this book gives many examples of these in dealing with the evolution of specific viral taxa.

Another important concept is that of quasi-species. RNA viruses have mutation rates of about $10^{-3}$ /nucleotide position/replication cycle which allows rapid changes in the genome - up to $1 \% /$ year in some viral populations, such as HIV-1. Consequently, it can be seen that a genome is not 'a defined unique structure but rather ... a weighted average of a large number of different individual sequences.' In this mixture of genomes, the quasi-species, the master sequence(s) can evolve rapidly (HIV-1) or remain static for long periods of time (Avian Flu A)

As in any compilation the quality of writing and ease of comprehension varies greatly between authors. Many important concepts are covered and it is a pity that this book falls between two readerships. It is too complex for the average reader with an interest in the subject and could well be out of date for the expert.

J. B. KURTZ

\section{PCR: Protocols for Diagnosis of Human and Animal Virus Diseases}

Edited by Y. BECKER and G. DARAI. 1995. ISBN 3-54058899-X. Springer-Verlag, Berlin. Pp. 596. DM 168.00.

The polymerase chain reaction (PCR) has become firmly established in all branches of molecular biology and has revolutionised the laboratory diagnosis of infectious disease, particularly those caused by viruses. Theoretically any infectious agent can be detected and characterised by PCR (with the possible exception of prion-associated encephalopathies) provided the nucleotide base sequence of a conserved (i.e., evolutionary stable) region of the agent's genome. is known.

This spring-bound book is divided into three sections. The first describes around 50 protocols for the detection and characterisation of viruses important in human disease. Part II gives 12 protocols of particular interest to those who care for livestock. A final, short section describes improvements in DNA and RNA preparation for PCR and the use of personal computer software in the design of PCR protocols.

This is a multi-author book with over 200 contributors. Therefore, there is some repetition and inconsistency of style, but this is minimal. It is not written for a PCR novice, and assumes full knowledge of PCR basics, such as oligonucleotide primers and thermal cycling. It is an exceptionally useful book that will soon become dog-eared in any molecular biology laboratory. But, included with the book is a computer diskette containing the whole book as document files. The publishers should be commended for such rare courtesy.

\section{G. BEARDS}

\section{Infection and Immunity}

J. Playfair. 1995. ISBN 0-19-854926-1. Oxford University Press. Pp. 154. Hardback $£ 20.00$, paperback $£ 9.95$.

At first glance, this book appears as a yet another book on 'Immunity to Infection'. However, this one is enjoyable to read and simple enough for even an 'interested lay person' to be able to understand it. Indeed, this is one readership population among many targeted by the author who presupposes no prior knowledge of microbiology or immunology. The targeted readership also includes students and potential students of science and medicine. Thus, as one would expect, a more informed graduate of science may find it a little simple.

The book consists of 20 short chapters that cover the important aspects of immunology, including natural and adaptive immune responses to pathogens and self antigens, the cellular basis of the humoral response and the functional attributes of the various cellular components of the immune system. In a series of lecture-style tutorials, the basic mechanisms of non-specific defence, antigen processing and presentation, immune-evasion by pathogens, immunodeficiency and autoimmunity are explained.

However, for a strict beginner a few more extensive graphical illustrations would probably have made the book even more enjoyable. The appendix consists of 10 selected infections which are discussed only briefly; this section could have been further expanded to accommodate more infections in slightly greater detail. Also, considering the target readership, the list of recommended books for further reading should have included more suitable books; for example, instead of Topley and Wilson's or Mandell's intimidating reference books, the more student-orientated medical microbiology text-book by Greenwood, Slack and Peutherer would have been more appropriate.

I certainly think there is a niche for this book among the specified target readership (i.e., beginners) and, therefore, would recommend it to first or second year students of microbiology and medicine.

D. Ala'AldeEn

\section{Oral Cephalosporins Volume 47 Antibiotics and Chemotherapy}

Edited by R. C. Moellering. 1995. ISBN 3-8055-6163-6.

S Karger AG, Basel. Pp. 189. SFr. 216.00.

Is there a need for a book exclusively devoted to oral cephalosporins? The answer to this surely depends, in part, on whether one believes that there is a need for oral cephalosporins in the first place, and I hope that it is not too dismissive to suggest that this is far from being intuitively obvious. Certainly there are few, if any, infective conditions requiring oral therapy in which cephalosporins represent the natural first choice. The earlier compounds, like cephalexin and its almost identical congener, cephradine, exhibit unimpressive intrinsic activity and are relatively slowly bactericidal, as their affinity for the penicillin-binding protein (PBP) targets is restricted to PBP-3. The newer ones, such as cefixime and ceftibuten have important gaps in their spectrum that seriously impede their usefulness for infections in which oral therapy is appropriate. Although cefdinir and the oral cephalosporin esters are generally broader in spectrum, they still lack useful activity against enterococci, pseudomonads and bacteroides; furthermore, parenteral administration is often more appropriate when broad-spectrum therapy embracing opportunist gram-negative rods is felt to be necessary.

The contributors to this volume in the Antibiotics and Chemotherapy series have no such reservations, and if you 